Mathematical Modelling AND ANALysis

Volume 17 Number 5, November 2012, 630-641 http://dx.doi.org/10.3846/13926292.2012.734864

(c) Vilnius Gediminas Technical University, 2012
Publisher: Taylor\&Francis and VGTU

http://www.tandfonline.com/TMMA

Print ISSN: 1392-6292

Online ISSN: 1648-3510

\title{
Classical Solution of the Cauchy Problem for Biwave Equation: Application of Fourier Transform
}

\author{
Victor Korzyuk ${ }^{a}$, Nguyen Van $\operatorname{Vinh}^{b}$ and \\ Nguyen Tuan Minh ${ }^{a}$ \\ ${ }^{a}$ Belarusian State University \\ 4, Fr. Scorina Ave., 220030 Minsk, Belarus \\ ${ }^{b}$ Hue University of Education \\ 34, Le Loi, 530000 Hue, Vietnam \\ E-mail(corresp.): korzyuk@bsu.by \\ E-mail: vinhnguyen0109@gmail.com; minhnguyen@yandex.ru
}

Received December 22, 2010; revised August 20, 2012; published online November 1, 2012

\begin{abstract}
In this paper, we use some Fourier analysis techniques to find an exact solution to the Cauchy problem for the $n$-dimensional biwave equation in the upper
\end{abstract} half-space $\mathbb{R}^{n} \times[0,+\infty)$.

Keywords: biwave equation, Fourier transform, Cauchy problem.

AMS Subject Classification: 35G05; 35G10.

\section{Introduction}

The Cauchy initial value problem for the $n$-dimensional biwave equation consists in finding a scalar function $u \in C^{4}\left(\mathbb{R}^{n} \times[0,+\infty)\right)$ such that for $(x, t) \in$ $\mathbb{R}^{n} \times(0,+\infty)$ then

$$
\left(\frac{\partial^{2}}{\partial t^{2}}-a^{2} \Delta\right)\left(\frac{\partial^{2}}{\partial t^{2}}-b^{2} \Delta\right) u(x, t)=f(x, t), \quad a^{2}>b^{2}>0,
$$

together with the initial conditions

$$
u(x, 0)=\phi_{0}(x), \quad \frac{\partial u}{\partial t}(x, 0)=\phi_{1}(x), \quad \frac{\partial^{2} u}{\partial t^{2}}(x, 0)=\phi_{2}(x), \quad \frac{\partial^{3} u}{\partial t^{3}}(x, 0)=\phi_{3}(x),
$$

for $(x, t) \in \mathbb{R}^{n} \times\{0\}$.

The biwave equation has been studied in some models related to the mathematical theory of elasticity. Let us consider the mathematical formulation for the displacement equation of a homogeneous isotropic elastic body. Remark 
that, the Newton's second law leads to the Cauchy's motion equation of an elastic body, which takes the form

$$
\nabla \cdot \boldsymbol{\sigma}+\mathbf{f}=\rho \ddot{\mathbf{u}}
$$

where $\boldsymbol{\sigma}$ is the Cauchy stress tensor field, $\mathbf{u}$ is the displacement vector field, $\mathbf{f}$ is the vector field of body force per unit volume and $\rho$ is the mass density.

The infinitesimal strain tensor field is given by the equation

$$
\varepsilon=\frac{1}{2}\left[\nabla \mathbf{u}+(\nabla \mathbf{u})^{T}\right]
$$

Moreover, the Hooke's law for homogeneous isotropic bodies has the form

$$
\boldsymbol{\sigma}=\lambda \operatorname{trace}(\varepsilon) \mathbf{I}+2 \mu \varepsilon,
$$

where $\lambda, \mu>0$ are Lamé's parameters and $\mathbf{I}$ is the second-order identity tensor. Substituting the strain-displacement equation (1.4) and the Hooke's equation (1.5) into the equilibrium equation (1.3), we obtain the Navier's elastodynamic wave equation

$$
(\lambda+\mu) \nabla \operatorname{div}(\mathbf{u})+\mu \Delta \mathbf{u}+\mathbf{f}=\rho \ddot{\mathbf{u}} .
$$

This equation in the Cartesian coordinates has the form

$$
(\lambda+\mu) \frac{\partial}{\partial x_{k}}\left(\sum_{j=1}^{n} \frac{\partial u_{j}}{\partial x_{j}}\right)+\mu \Delta u_{k}+f_{k}=\rho \frac{\partial^{2} u_{k}}{\partial t^{2}}, \quad k=1, \ldots, n .
$$

Let us denote $a^{2}=(\lambda+2 \mu) / \rho, b^{2}=\mu / \rho$, then (1.6) can be rewritten as

$$
\mathcal{L} \equiv\left(\frac{\partial^{2}}{\partial t^{2}}-b^{2} \Delta\right) \mathbf{u}-\left(a^{2}-b^{2}\right) \nabla \operatorname{div}(\mathbf{u})-\frac{\mathbf{f}}{\rho}=0 .
$$

It is easy to show that the equation (1.7) has a solution in the following form

$$
\mathbf{u}=\left(\frac{\partial^{2}}{\partial t^{2}}-a^{2} \Delta\right) \mathbf{w}+\left(a^{2}-b^{2}\right) \nabla \operatorname{div}(\mathbf{w}),
$$

where $\mathbf{w}$ is a solution to the biwave equation

$$
\left(\frac{\partial^{2}}{\partial t^{2}}-a^{2} \Delta\right)\left(\frac{\partial^{2}}{\partial t^{2}}-b^{2} \Delta\right) \mathbf{w}=\frac{\mathbf{f}}{\rho} .
$$

This formula is called as Cauchy-Kovalevski-Somigliana solution to the elastodynamic wave equation. Indeed, substituting (1.8)-(1.9) to the left-hand side of (1.7), we have

$$
\begin{aligned}
\mathcal{L}= & \left(\frac{\partial^{2}}{\partial t^{2}}-b^{2} \Delta\right)\left(\left(\frac{\partial^{2}}{\partial t^{2}}-a^{2} \Delta\right) \mathbf{w}+\left(a^{2}-b^{2}\right) \nabla \operatorname{div}(\mathbf{w})\right) \\
& -\left(a^{2}-b^{2}\right) \nabla\left(\left(\frac{\partial^{2}}{\partial t^{2}}-a^{2} \Delta\right) \operatorname{div}(\mathbf{w})+\left(a^{2}-b^{2}\right) \Delta \operatorname{div}(\mathbf{w})\right)-\frac{\mathbf{f}}{\rho} .
\end{aligned}
$$


Note that

$$
\left(\frac{\partial^{2}}{\partial t^{2}}-a^{2} \Delta\right) \operatorname{div}(\mathbf{w})+\left(a^{2}-b^{2}\right) \Delta \operatorname{div}(\mathbf{w})=\left(\frac{\partial^{2}}{\partial t^{2}}-b^{2} \Delta\right) \operatorname{div}(\mathbf{w}) .
$$

Therefore, we get

$$
\begin{aligned}
\mathcal{L}= & \left(\frac{\partial^{2}}{\partial t^{2}}-b^{2} \Delta\right)\left(\frac{\partial^{2}}{\partial t^{2}}-a^{2} \Delta\right) \mathbf{w}+\left(a^{2}-b^{2}\right)\left(\frac{\partial^{2}}{\partial t^{2}}-b^{2} \Delta\right) \nabla \operatorname{div}(\mathbf{w}) \\
& -\left(a^{2}-b^{2}\right) \nabla\left(\frac{\partial^{2}}{\partial t^{2}}-b^{2} \Delta\right) \operatorname{div}(\mathbf{w})-\frac{\mathbf{f}}{\rho}=0 .
\end{aligned}
$$

For more explanations about physical context, we refer the reader to $[5$, $8,10]$. Actually, there are not many mathematical papers related to biwave equations because it gets more difficult when studying high-order PDEs. In some recent researches, the symmetry analysis of biwave equations is considered and exact solutions are obtained by Fushchych, Roman and Zhdanov [3]; the existence and uniqueness of the solution to Cauchy initial value problem, bounded valued problem are given by Korzyuk, Cheb and Konopelko [6, 7]; the finite element methods for approximations of biwave equation are developed by Feng and Neilan $[1,2]$. In our present work, the main result is to show the exact classical solution to the Cauchy initial value problem for the $n$-dimensional biwave equation by using some techniques of Fourier analysis.

Returning to the Cauchy problem for the biwave equation (1.1), we suppose that $\phi_{0}, \phi_{1}, \phi_{2}, \phi_{3}$, and $f$ are elements in Schwartz space $\mathcal{S}\left(\mathbb{R}^{n}\right)$ of rapidly decreasing functions on $\mathbb{R}^{n}$. Remark that, an indefinitely differentiable function $\phi$ is called rapidly decreasing when $\phi$ and all its derivatives are required to satisfy that

$$
\|\phi\|_{\alpha, \beta}=\sup _{x \in \mathbb{R}^{n}}\left|x^{\alpha}\left(\frac{\partial}{\partial x}\right)^{\beta} \phi(x)\right|<\infty
$$

for every multi-index $\alpha$ and $\beta$. The Fourier transform of Schwartz function $\phi \in \mathcal{S}\left(\mathbb{R}^{n}\right)$ is defined by

$$
\mathcal{F}[\phi](\xi) \equiv \widehat{\phi}(\xi)=\int_{\mathbb{R}^{n}} e^{-i x \cdot \xi} \phi(x) d x .
$$

The convolution of two integrable functions $\phi$ and $\psi$ is written as $\phi * \psi$. It is defined as the integral of the product of the two functions after one is reversed and shifted. As such, it is a particular kind of integral transform:

$$
(\phi * \psi)(t)=\int_{\mathbb{R}^{n}} \phi(\tau) \psi(t-\tau) d \tau .
$$

In the Euclidean space $\mathbb{R}^{n}$, the spherical mean of an integrable function $\phi$ around a point $x$ is the average of all values of that function on a sphere of radius $R$ centered at that point, i.e. it is defined by the formula

$$
\mathcal{M}_{R}(\phi)(x)=\frac{1}{\omega_{n} R^{n-1}} \int_{\partial B(x, R)} \phi(y) d \sigma(y) \equiv \frac{1}{\omega_{n}} \int_{\partial B(0,1)} \phi(x+R y) d \sigma(y),
$$

where $\omega_{n}$ is the surface area of the $n$-dimensional unit ball and $\sigma$ is the spherical measure area. 


\section{Main Results}

The Cauchy problem for the homogeneous biwave equation in $\mathbb{R}^{n} \times[0,+\infty)$ that we will be studying in this section, reads as follows

$$
\left(\frac{\partial^{2}}{\partial t^{2}}-a^{2} \Delta\right)\left(\frac{\partial^{2} u}{\partial t^{2}}-b^{2} \Delta u\right)=0, \quad a^{2}>b^{2}>0
$$

with the initial conditions

$$
\left.u\right|_{t=0}=\phi_{0}(x),\left.\frac{\partial u}{\partial t}\right|_{t=0}=\phi_{1}(x),\left.\quad \frac{\partial^{2} u}{\partial t^{2}}\right|_{t=0}=\phi_{2}(x),\left.\quad \frac{\partial^{3} u}{\partial t^{3}}\right|_{t=0}=\phi_{3}(x),
$$

where $\phi_{0}, \phi_{1}, \phi_{2}, \phi_{3}$ are Schwartz functions.

The equation (2.1) can be rewritten as a fourth-order PDE, which has the following form

$$
\frac{\partial^{4} u}{\partial t^{4}}-\left(a^{2}+b^{2}\right) \frac{\partial^{2}}{\partial t^{2}} \Delta u+a^{2} b^{2} \Delta^{2} u=0
$$

Taking Fourier transform to the both sides of the equation (2.3), we obtain

$$
\frac{\partial^{4}}{\partial t^{4}} \widehat{u}(\xi, t)+\left(a^{2}+b^{2}\right)|\xi|^{2} \frac{\partial^{2}}{\partial t^{2}} \widehat{u}(\xi, t)+a^{2} b^{2}|\xi|^{4} \widehat{u}(\xi, t)=0
$$

This fourth ODE has the general solution, which takes the form

$$
\widehat{u}(\xi, t)=C_{1} \cos (a|\xi| t)+C_{2} \sin (a|\xi| t)+C_{3} \cos (b|\xi| t)+C_{4} \sin (b|\xi| t),
$$

where parameters $C_{1}, C_{2}, C_{3}, C_{4}$ are determined from the initial conditions:

$$
\begin{aligned}
\left.\widehat{u}(\xi, t)\right|_{t=0}= & C_{1}+C_{3}=\widehat{\phi_{0}}(\xi),\left.\quad \frac{\partial \widehat{u}(\xi, t)}{\partial t}\right|_{t=0}=a C_{2}|\xi|+b C_{4}|\xi|=\widehat{\phi_{1}}(\xi), \\
& \left.\frac{\partial^{2} \widehat{u}(\xi, 0)}{\partial t^{2}}\right|_{t=0}=-a^{2} C_{1}|\xi|^{2}-b^{2} C_{3}|\xi|^{2}=\widehat{\phi_{2}}(\xi), \\
& \left.\frac{\partial^{3} \widehat{u}(\xi, 0)}{\partial t^{3}}\right|_{t=0}=-a^{3} C_{2}|\xi|^{3}-b^{3} C_{4}|\xi|^{3}=\widehat{\phi_{3}}(\xi) .
\end{aligned}
$$

Solving above system of equations, we easily get the image of solution $u(x, t)$ via Fourier transform given by

$$
\begin{aligned}
\widehat{u}(\xi, t)= & -\frac{b^{2}|\xi|^{2} \widehat{\phi_{0}}(\xi)+\widehat{\phi_{2}}(\xi)}{\left(a^{2}-b^{2}\right)|\xi|^{2}} \cos (a|\xi| t)-\frac{b^{2}|\xi|^{2} \widehat{\phi_{1}}(\xi)+\widehat{\phi_{3}}(\xi)}{\left(a^{3}-a b^{2}\right)|\xi|^{3}} \sin (a|\xi| t) \\
& +\frac{a^{2}|\xi|^{2} \widehat{\phi_{0}}(\xi)+\widehat{\phi_{2}}(\xi)}{\left(a^{2}-b^{2}\right)|\xi|^{2}} \cos (b|\xi| t)+\frac{a^{2}|\xi|^{2} \widehat{\phi_{1}}(\xi)+\widehat{\phi_{3}}(\xi)}{\left(a^{2} b-b^{3}\right)|\xi|^{3}} \sin (b|\xi| t),
\end{aligned}
$$


or by the rewritten form

$$
\begin{aligned}
\widehat{u}(\xi, t)= & -\frac{b^{2}}{a^{2}-b^{2}} \widehat{\phi_{0}}(\xi) \cos (a|\xi| t)+\frac{a^{2}}{a^{2}-b^{2}} \widehat{\phi_{0}}(\xi) \cos (b|\xi| t) \\
& -\frac{b^{2}}{a\left(a^{2}-b^{2}\right)} \widehat{\phi_{1}}(\xi) \frac{\sin (a|\xi| t)}{|\xi|}+\frac{a^{2}}{b\left(a^{2}-b^{2}\right)} \widehat{\phi_{1}}(\xi) \frac{\sin (b|\xi| t)}{|\xi|} \\
& +\frac{\widehat{\phi_{3}}(\xi)}{a^{2}-b^{2}}\left[\frac{1}{b} \frac{\sin (b|\xi| t)}{|\xi|^{3}}-\frac{1}{a} \frac{\sin (a|\xi| t)}{|\xi|^{3}}\right] \\
& +\frac{\widehat{\phi_{2}}(\xi)}{a^{2}-b^{2}}\left[\frac{\cos (b|\xi| t)}{|\xi|^{2}}-\frac{\cos (a|\xi| t)}{|\xi|^{2}}\right] .
\end{aligned}
$$

In the next sequence, we will find the inverse formula of (2.4) and obtain an exact solution to the equation (2.1).

Theorem 1. The Cauchy problem for the homogeneous biwave equation in $\mathbb{R} \times$ $[0,+\infty)$ has the following solution

$$
\begin{aligned}
u(x, t)= & \frac{1}{2 a b\left(a^{2}-b^{2}\right)}\left[-b^{3} \int_{x-a t}^{x+a t} \phi_{1}(y) d y+a^{3} \int_{x-b t}^{x+b t} \phi_{1}(y) d y\right. \\
& -a b \int_{x-a t}^{x-b t} \int_{0}^{y} \phi_{2}(u) d u d y+a b \int_{x+b t}^{x+a t} \int_{0}^{y} \phi_{2}(u) d u d y \\
& +b \int_{x-a t}^{x+a t} \int_{0}^{y} \int_{0}^{\tau+b t} \phi_{3}(\omega) d \omega d \tau d y-a \int_{x-b t}^{y} \int_{0}^{\tau} \int_{0}^{\tau} \phi_{3}(\omega) d \omega d \tau d y \\
& \left.-a b^{3} \phi_{0}(x+a t)-a b^{3} \phi_{0}(x-a t)+a^{3} b \phi_{0}(x+b t)+a^{3} b \phi_{0}(x-b t)\right] .
\end{aligned}
$$

Proof. We have that

$$
\begin{aligned}
\cos (a|\xi| t) & =\frac{e^{i a|\xi| t}+e^{-i a|\xi| t}}{2}, \quad \sin (a|\xi| t)=\frac{e^{i a|\xi| t}-e^{-i a|\xi| t}}{2 i} \\
\frac{\sin (a|\xi| t)}{|\xi|} & =\frac{e^{i a|\xi| t}-e^{-i a|\xi| t}}{2 i|\xi|}=\frac{1}{2} \int_{-a t}^{a t} e^{i|\xi| \theta} d \theta \\
\frac{\cos (a|\xi| t)}{|\xi|^{2}} & =\frac{e^{i a|\xi| t}+e^{-i a|\xi| t}}{2|\xi|^{2}} \\
& =-\frac{1}{2} \int_{0}^{a t} \int_{0}^{y} e^{i|\xi| u} d u d y-\frac{1}{2} \int_{0}^{a t} \int_{0}^{y} e^{-i|\xi| u} d u d y+\frac{1}{|\xi|^{2}} \\
\frac{\sin (a|\xi| t)}{|\xi|^{3}} & =\frac{e^{i a|\xi| t}-e^{-i a|\xi| t}}{2 i|\xi|^{3}}=-\frac{1}{2} \int_{-a t}^{a t} \int_{0}^{y} \int_{0}^{\tau} e^{i|\xi| u} d u d \tau d y+\frac{a t}{|\xi|^{2}}
\end{aligned}
$$

Moreover,

$$
\widehat{\delta}(x-\alpha t)=\int_{-\infty}^{+\infty} e^{-i|\xi| x} \delta(x-\alpha t) d x=e^{-i \alpha|\xi| t}
$$


where $\delta(x)$ is the Dirac delta function. Hence, by the property of Dirac's delta function, we note that

$$
\begin{aligned}
& \left(\frac{e^{i a|\xi| t}+e^{-i a|\xi| t}}{2}\right) \widehat{\phi_{0}}(\xi)=\frac{\mathcal{F}\left[\left(\delta(x+a t) * \phi_{0}(x)\right)\right]+\mathcal{F}\left[\left(\delta(x-a t) * \phi_{0}(x)\right)\right]}{2}, \\
& \widehat{\phi_{1}}(\xi)\left(\frac{1}{2} \int_{-a t}^{a t} e^{i|\xi| \theta} d \theta\right)=\frac{1}{2} \int_{-a t}^{a t} \mathcal{F}\left[\delta(x+\theta) * \phi_{1}(x)\right] d \theta, \\
& \widehat{\phi_{2}}(\xi)\left(-\frac{1}{2} \int_{0}^{a t} \int_{0}^{y} e^{i|\xi| u} d u d y-\frac{1}{2} \int_{0}^{a t} \int_{0}^{y} e^{-i|\xi| u} d u d y\right) \\
& =-\frac{1}{2}\left(\int_{0}^{a t} \int_{0}^{y} \mathcal{F}\left[\delta(x+u) * \phi_{2}(x)\right] d u d y+\int_{0}^{a t} \int_{0}^{y} \mathcal{F}\left[\delta(x-u) * \phi_{2}(x)\right] d u d y\right), \\
& \widehat{\phi_{3}}(\xi)\left(\int_{a t}^{a t} \int_{0}^{y} \int_{0}^{\tau} e^{i|\xi| u} d u d \tau d y\right)=\int_{-a t}^{y} \int_{0}^{\tau} \int_{0}^{\tau} \mathcal{F}\left[\delta(x+u) * \phi_{3}(x)\right] d u d \tau d y .
\end{aligned}
$$

Substituting the above identities into the formula (2.4), we obtain that

$$
\begin{aligned}
\widehat{u}(\xi, t)= & -\frac{b^{2}}{\left(a^{2}-b^{2}\right)} \frac{\mathcal{F}\left[\delta(x+a t) * \phi_{0}(x)\right]+\mathcal{F}\left[\delta(x-a t) * \phi_{0}(x)\right]}{2} \\
& +\frac{a^{2}}{\left(a^{2}-b^{2}\right)} \frac{\mathcal{F}\left[\delta(x+b t) * \phi_{0}(x)\right]+\mathcal{F}\left[\delta(x-b t) * \phi_{0}(x)\right]}{2} \\
& -\frac{b^{2}}{\left(a^{3}-a b^{2}\right)} \frac{1}{2} \int_{-a t}^{a t} \mathcal{F}\left[\delta(x+\theta) * \phi_{1}(x)\right] d \theta \\
& +\frac{a^{2}}{\left(a^{2} b-b^{3}\right)} \frac{1}{2} \int_{-b t}^{b t} \mathcal{F}\left[\delta(x+\theta) * \phi_{1}(x)\right] d \theta \\
& -\frac{1}{\left(a^{2}-b^{2}\right)}\left(-\frac{1}{2} \int_{0}^{a t} \int_{0}^{y} \mathcal{F}\left[\delta(x+u) * \phi_{2}(x)\right] d u d y\right. \\
& \left.-\frac{1}{2} \int_{0}^{a t} \int_{0}^{y} \mathcal{F}\left[\delta(x-u) * \phi_{2}(x)\right] d u d y\right) \\
& +\frac{1}{\left(a^{2}-b^{2}\right)}\left(-\frac{1}{2} \int_{0}^{b t} \int_{0}^{y} \mathcal{F}\left[\delta(x+u) * \phi_{2}(x)\right] d u d y\right. \\
& \left.-\frac{1}{2} \int_{0}^{b t} \int_{0}^{y} \mathcal{F}\left[\delta(x-u) * \phi_{2}(x)\right] d u d y\right) \\
& +\frac{1}{\left(a^{3}-a b^{2}\right)} \frac{1}{2} \int_{-a t}^{a t} \int_{0}^{y} \int_{0}^{\tau} \mathcal{F}\left[\delta(x+u) * \phi_{3}(x)\right] d u d \tau d y \\
& -\frac{1}{\left(a^{2} b-b^{3}\right)} \frac{1}{2} \int_{-b t}^{b t} \int_{0}^{y} \int_{0}^{\tau} \mathcal{F}\left[\delta(x+u) * \phi_{3}(x)\right] d u d \tau d y .
\end{aligned}
$$

Consequently, we get the inverse formula of $\widehat{u}$ given by

$$
u(x, t)=-\frac{b^{2}}{\left(a^{2}-b^{2}\right)} \frac{\left(\delta(x+a t) * \phi_{0}(x)\right)+\left(\delta(x-a t) * \phi_{0}(x)\right)}{2}
$$




$$
\begin{aligned}
& +\frac{a^{2}}{\left(a^{2}-b^{2}\right)} \frac{\left(\delta(x+b t) * \phi_{0}(x)\right)+\left(\delta(x-b t) * \phi_{0}(x)\right)}{2} \\
& -\frac{b^{2}}{\left(a^{3}-a b^{2}\right)} \frac{1}{2} \int_{-a t}^{a t}\left(\delta(x+\theta) * \phi_{1}(x)\right) d \theta \\
& +\frac{a^{2}}{\left(a^{2} b-b^{3}\right)} \frac{1}{2} \int_{-b t}^{b t}\left(\delta(x+\theta) * \phi_{1}(x)\right) d \theta \\
& -\frac{1}{\left(a^{2}-b^{2}\right)}\left(-\frac{1}{2} \int_{0}^{a t} \int_{0}^{y}\left(\delta(x+u) * \phi_{2}(x)\right) d u d y\right. \\
& \left.-\frac{1}{2} \int_{0}^{a t} \int_{0}^{y}\left(\delta(x-u) * \phi_{2}(x)\right) d u d y\right) \\
& +\frac{1}{\left(a^{2}-b^{2}\right)}\left(-\frac{1}{2} \int_{0}^{b t} \int_{0}^{y}\left(\delta(x+u) * \phi_{2}(x)\right) d u d y\right. \\
& \left.-\frac{1}{2} \int_{0}^{b t} \int_{0}^{y}\left(\delta(x-u) * \phi_{2}(x)\right) d u d y\right) \\
& +\frac{1}{\left(a^{3}-a b^{2}\right)} \frac{1}{2} \int_{-a t}^{a t} \int_{0}^{y} \int_{0}^{\tau}\left(\delta(x+u) * \phi_{3}(x)\right) d u d \tau d y \\
& -\frac{1}{\left(a^{2} b-b^{3}\right)} \frac{1}{2} \int_{-b t}^{b t} \int_{0}^{y} \int_{0}^{\tau}\left(\delta(x+u) * \phi_{3}(x)\right) d u d \tau d y .
\end{aligned}
$$

The last formula is equivalent to the one given at (2.5), so the theorem is proved.

For the generalized case, we will use the following result:

Lemma 1. For an odd number $n \geq 3, m=\frac{n-3}{2}$ and $0 \leq k \leq m$ then

$$
\int_{-R}^{R} e^{i s|\xi|}\left(R^{2}-s^{2}\right)^{m-k} d s=\frac{1}{2^{k} k !}\left(\frac{1}{R} \frac{\partial}{\partial R}\right)^{k}\left(\frac{1}{\omega_{n-1} R} \int_{\partial B(0, R)} e^{-i x \cdot \xi} d \sigma(x)\right) .
$$

For the proof, we refer the reader to Torchinsky's paper in [12]. Note that, in the case $k=m$, it follows that

$$
\begin{aligned}
\frac{\sin (a|\xi| t)}{|\xi|} & =\frac{1}{2} \int_{-a t}^{a t} e^{i s|\xi|} d s \\
& =\frac{1}{2^{m+1} m !}\left(\frac{1}{a^{2} t} \frac{\partial}{\partial t}\right)^{m}\left(\frac{1}{\omega_{n-1} a t} \int_{\partial B(0, a t)} e^{-i x \cdot \xi} d \sigma(x)\right)
\end{aligned}
$$

Differentiating with respect to $t$, then

$$
\cos (a|\xi| t)=\frac{1}{2^{m+1} m ! a} \frac{\partial}{\partial t}\left(\frac{1}{a^{2} t} \frac{\partial}{\partial t}\right)^{m}\left(\frac{1}{\omega_{n-1} a t} \int_{\partial B(0, a t)} e^{-i x \cdot \xi} d \sigma(x)\right)
$$


On the other hand, we have

$$
\begin{gathered}
\frac{\cos (b|\xi| t)}{|\xi|^{2}}-\frac{\cos (a|\xi| t)}{|\xi|^{2}}=\int_{b t}^{a t} \frac{\sin (s|\xi|)}{|\xi|} d s=\frac{1}{2} \int_{b t}^{a t} \int_{-s}^{s} e^{i \tau|\xi|} d \tau d s \\
=\int_{b t}^{a t} \frac{1}{2^{m+1} m !}\left(\frac{1}{s} \frac{\partial}{\partial s}\right)^{m}\left(\frac{1}{\omega_{n-1} s} \int_{\partial B(0, s)} e^{-i x \cdot \xi} d \sigma(x)\right) d s .
\end{gathered}
$$

Integrating the above formula with respect to $t$, it implies that

$$
\begin{aligned}
& \frac{1}{b} \frac{\sin (b|\xi| t)}{|\xi|^{3}}-\frac{1}{a} \frac{\sin (a|\xi| t)}{|\xi|^{3}}=\frac{1}{2} \int_{0}^{t} \int_{b \nu}^{a \nu} \int_{-s}^{s} e^{i \tau|\xi|} d \tau d s d \nu \\
& \quad=\int_{0}^{t} \int_{b \nu}^{a \nu} \frac{1}{2^{m+1} m !}\left(\frac{1}{s} \frac{\partial}{\partial s}\right)^{m}\left(\frac{1}{\omega_{n-1} s} \int_{\partial B(0, s)} e^{-i x \cdot \xi} d \sigma(x)\right) d s d \nu
\end{aligned}
$$

Moreover, for each function $\theta \in \mathcal{S}\left(\mathbb{R}^{n}\right)$, we also have

$$
\begin{aligned}
& \int_{\mathbb{R}^{n}} \frac{\sin (a|\xi| t)}{|\xi|} \theta(\xi) d \xi=\frac{1}{2^{m+1} m !}\left(\frac{1}{a^{2} t} \frac{\partial}{\partial t}\right)^{m} \frac{1}{\omega_{n-1} a t} \\
& \times \int_{\partial B(0, a t) \mathbb{R}^{n}} \int^{-i x \cdot \xi} \theta(\xi) d \xi d \sigma(x)=\frac{1}{2^{m+1} m !}\left(\frac{1}{a^{2} t} \frac{\partial}{\partial t}\right)^{m} \frac{1}{\omega_{n-1} a t} \int_{\partial B(0, a t)} \hat{\theta}(x) d \sigma(x) .
\end{aligned}
$$

Therefore, we conclude that

$$
\frac{1}{2^{m+1} m !}\left(\frac{1}{a^{2} t} \frac{\partial}{\partial t}\right)^{m}\left(\frac{1}{\omega_{n-1} a t} \int_{\partial B(0, a t)} d \sigma(x)\right) \quad(\xi)=\frac{\sin (a|\xi| t)}{|\xi|} .
$$

By the Fourier inversion and convolution formulas, we obtain the identity

$$
\begin{aligned}
& \frac{1}{(2 \pi)^{n}} \int_{\mathbb{R}^{n}} \widehat{\phi_{1}}(\xi) \frac{\sin (a|\xi| t)}{|\xi|} e^{i \xi \cdot x} d \xi \\
& =\frac{1}{2^{m+1} m !}\left(\frac{1}{a^{2} t} \frac{\partial}{\partial t}\right)^{m}\left(\frac{1}{\omega_{n-1} a t} \int_{\partial B(x, a t)} \phi_{1}(y) d \sigma(y)\right) \\
& =\frac{\omega_{n}}{2^{m+1} m ! \omega_{n-1}}\left(\frac{1}{a^{2} t} \frac{\partial}{\partial t}\right)^{m}\left((a t)^{n-2} M_{a t}\left(\phi_{1}\right)(x)\right) \\
& =\frac{1}{(n-2) ! !}\left(\frac{1}{a^{2} t} \frac{\partial}{\partial t}\right)^{m}\left((a t)^{n-2} M_{a t}\left(\phi_{1}\right)(x)\right) .
\end{aligned}
$$

Applying the same way for the expressions (2.7)-(2.9) and substituting the obtained identities into the formula $(2.4)$, we have found an exact solution to the $n$-dimensional biwave equation, where $n \geq 3$ is an odd number: 
Theorem 2. The Cauchy initial value problem for the homogeneous n-dimensional biwave equation, where $n \geq 3$ is an odd number, has the following solution

$$
\begin{aligned}
u(x, t) & =\frac{1}{(n-2) ! !\left(a^{2}-b^{2}\right)}\left[\frac{a^{2}}{b} \frac{\partial}{\partial t}\left(\frac{1}{b^{2} t} \frac{\partial}{\partial t}\right)^{\frac{n-3}{2}}\left((b t)^{n-2} M_{b t}\left(\phi_{0}\right)(x)\right)\right. \\
& -\frac{b^{2}}{a} \frac{\partial}{\partial t}\left(\frac{1}{a^{2} t} \frac{\partial}{\partial t}\right)^{\frac{n-3}{2}}\left((a t)^{n-2} M_{a t}\left(\phi_{0}\right)(x)\right)+\frac{a^{2}}{b}\left(\frac{1}{b^{2} t} \frac{\partial}{\partial t}\right)^{\frac{n-3}{2}} \\
& \times\left((b t)^{n-2} M_{b t}\left(\phi_{1}\right)(x)\right)-\frac{b^{2}}{a}\left(\frac{1}{a^{2} t} \frac{\partial}{\partial t}\right)^{\frac{n-3}{2}}\left((a t)^{n-2} M_{a t}\left(\phi_{1}\right)(x)\right) \\
& +\int_{b t}^{a t}\left(\frac{1}{s} \frac{\partial}{\partial s}\right)^{\frac{n-3}{2}}\left((s)^{n-2} M_{s}\left(\phi_{2}\right)(x)\right) d s \\
& \left.+\int_{0}^{t} \int_{b \nu}^{a \nu}\left(\frac{1}{s} \frac{\partial}{\partial s}\right)^{\frac{n-3}{2}}\left((s)^{n-2} M_{s}\left(\phi_{3}\right)(x)\right) d s d \nu\right] .
\end{aligned}
$$

Now we consider the case when $n$ is an even number. The Hadamard's method of descent (see e.g. [4]) is useful to connect with the case in the odd dimensional space $\mathbb{R}^{n+1}$. For fixed $T>0$, we choose a Schwartz function $\eta \in \mathcal{S}(\mathbb{R})$, such that $\eta\left(x_{n+1}\right)=1$ for all $\left|x_{n+1}\right| \leq n T$. Let us denote

$$
\overline{\phi_{i}}\left(x_{1}, x_{2}, \ldots, x_{n}, x_{n+1}\right)=\phi_{i}\left(x_{1}, x_{2}, \ldots, x_{n}\right) \eta\left(x_{n+1}\right), \quad i=0,1,2,3 .
$$

It is easy to see that $\overline{\phi_{i}} \in \mathcal{S}\left(\mathbb{R}^{n+1}\right)$. For $\left|x_{n+1}\right| \leq T, t \leq T$, the solution $\bar{u}\left(x_{1}, x_{2}, \ldots, x_{n+1}, t\right)$ to the Cauchy problem for $(n+1)$-dimensional biwave equation with initial valued functions $\overline{\phi_{i}}, i=0,1,2,3$ does not depend on $x_{n+1}$. In particular,

$$
u\left(x_{1}, x_{2}, \ldots, x_{n}, t\right)=\bar{u}\left(x_{1}, x_{2}, \ldots, x_{n}, 0, t\right)
$$

is the solution to the $n$-dimensional wave equation for all $|t| \leq T$. Since $T$ is arbitrary, so $u$ is the solution to the Cauchy problem in even dimensional space $\mathbb{R}^{n}$.

Lemma 2. Given a function $f: \mathbb{R}^{n+1} \rightarrow \mathbb{R}$, which does not depend on the last variable, i.e. $f\left(x_{1}, x_{2}, \ldots, x_{n+1}\right)=g\left(x_{1}, x_{2}, \ldots, x_{n}\right)$, then

$$
\mathcal{M}_{t}(f)(x, 0)=\frac{2}{\omega_{n+1}} \int_{B_{n}(0,1)} \frac{g(x+t z)}{\sqrt{1-|z|^{2}}} d z .
$$

Proof. Observe that, for $\widetilde{x}=(x, 0)$ and $\widetilde{y}=\left(y, y_{n+1}\right)$, we have

$$
\mathcal{M}_{t}(f)(\widetilde{x})=\frac{1}{\omega_{n+1}} \int_{\partial B_{n+1}(0,1)} f(\widetilde{x}+t \widetilde{y}) d \sigma(\widetilde{y}) .
$$

We use the spherical coordinates given by

$$
\left\{\begin{array}{l}
y_{1}=\sin \varphi_{1} \sin \varphi_{2} \cdots \sin \varphi_{n-2} \sin \varphi_{n-1} \sin \varphi_{n} \\
y_{2}=\sin \varphi_{1} \sin \varphi_{2} \cdots \sin \varphi_{n-2} \sin \varphi_{n-1} \cos \varphi_{n}
\end{array}\right.
$$




$$
\left\{\begin{array}{l}
y_{3}=\sin \varphi_{1} \sin \varphi_{2} \cdots \sin \varphi_{n-2} \cos \varphi_{n-1} \cos \varphi_{n} \\
\cdots \\
y_{n}=\sin \varphi_{1} \cos \varphi_{2}, \quad y_{n+1}=\cos \varphi_{1}
\end{array}\right.
$$

where $0 \leq \varphi_{k} \leq \pi, k=1,2, \ldots, n-1$ and $0 \leq \varphi_{n} \leq 2 \pi$. The Jacobian of this transformation is calculated as

$$
J=\sin ^{n-1} \varphi_{1} \sin ^{n-2} \varphi_{2} \cdots \sin \varphi_{n-1} .
$$

Therefore

$$
\mathcal{M}_{t}(f)(x, 0)=\frac{1}{\omega_{n+1}} \int_{0}^{\pi} \ldots \int_{0}^{\pi} \int_{0}^{2 \pi} g(x+t y) J d \varphi_{1} d \varphi_{2} \ldots d \varphi_{n} .
$$

Let us give $r=\sin \varphi_{1}$, and

$$
\left\{\begin{array}{l}
z_{1}=r \sin \varphi_{2} \cdots \sin \varphi_{n-1} \sin \varphi_{n} \\
z_{2}=r \sin \varphi_{2} \cdots \sin \varphi_{n-1} \cos \varphi_{n} \\
\cdots \\
z_{n}=r \cos \varphi_{2} .
\end{array}\right.
$$

The Jacobian of above transformation is calculated by the formula

$$
J^{\prime}=\frac{1}{r^{n-1} \sin ^{n-2} \varphi_{2} \sin ^{n-3} \varphi_{3} \cdots \sin \varphi_{n-1}} .
$$

Finally, we obtain that

$$
\begin{aligned}
\mathcal{M}_{t}(f)(x, 0) & =\frac{2}{\omega_{n+1}} \int_{0}^{\pi} \ldots \int_{0}^{\pi} \int_{0}^{2 \pi} \int_{0}^{1} g(x+t z) \frac{1}{\cos \varphi_{1}} J d r d \varphi_{2} \ldots d \varphi_{n} \\
& =\frac{2}{\omega_{n+1}} \int_{0}^{\pi} \ldots \int_{0}^{\pi} \int_{0}^{2 \pi} \int_{0}^{1} g(x+t z) \frac{1}{\sqrt{1-|z|^{2}}} J d r d \varphi_{2} \ldots d \varphi_{n} \\
& =\frac{2}{\omega_{n+1}} \int_{0}^{\pi} \ldots \int_{0}^{\pi} \int_{0}^{2 \pi} \int_{0}^{1} g(x+t z) \frac{1}{\sqrt{1-|z|^{2}}} J J^{\prime} d z_{1} d z_{2} \ldots d z_{n} \\
& =\frac{2}{\omega_{n+1}} \int_{B_{n}(0,1)} \frac{g(x+t z)}{\sqrt{1-|z|^{2}}} d z .
\end{aligned}
$$

So the lemma is proved.

We use the notation $\widetilde{\mathcal{M}}_{t}(f)(x)=\frac{2}{\omega_{n+1}} \int_{B_{n}(0,1)} \frac{f(x+t z)}{\sqrt{1-|z|^{2}}} d z$ for a modified spherical mean of $f$ (see e.g. $[9,11]$ ). Applying the result of Lemma 2, we obtain the formula of the solution to the biwave equation in the even dimensional space $\mathbb{R}^{n}$ :

Theorem 3. The Cauchy initial value problem for the homogeneous n-dimensional biwave equation, where $n \geq 2$ is an even number, has the following solution

$$
\begin{aligned}
u(x, t)= & \frac{1}{(n-1) ! !\left(a^{2}-b^{2}\right)}\left[\frac{a^{2}}{b} \frac{\partial}{\partial t}\left(\frac{1}{b^{2} t} \frac{\partial}{\partial t}\right)^{\frac{n-2}{2}}\left((b t)^{n-1} \widetilde{\mathcal{M}}_{b t}\left(\phi_{0}\right)(x)\right)\right. \\
& -\frac{b^{2}}{a} \frac{\partial}{\partial t}\left(\frac{1}{a^{2} t} \frac{\partial}{\partial t}\right)^{\frac{n-2}{2}}\left((a t)^{n-1} \widetilde{\mathcal{M}}_{a t}\left(\phi_{0}\right)(x)\right)
\end{aligned}
$$




$$
\begin{aligned}
& +\frac{a^{2}}{b}\left(\frac{1}{b^{2} t} \frac{\partial}{\partial t}\right)^{\frac{n-2}{2}}\left((b t)^{n-1} \widetilde{\mathcal{M}}_{b t}\left(\phi_{1}\right)(x)\right)-\frac{b^{2}}{a}\left(\frac{1}{a^{2} t} \frac{\partial}{\partial t}\right)^{\frac{n-2}{2}} \\
& \times\left((a t)^{n-1} \widetilde{\mathcal{M}}_{a t}\left(\phi_{1}\right)(x)\right)+\int_{b t}^{a t}\left(\frac{1}{s} \frac{\partial}{\partial s}\right)^{\frac{n-2}{2}}\left((s)^{n-1} \widetilde{\mathcal{M}}_{s}\left(\phi_{2}\right)(x)\right) d s \\
& \left.-\int_{0}^{t} \int_{b \nu}^{a \nu}\left(\frac{1}{s} \frac{\partial}{\partial s}\right)^{\frac{n-2}{2}}\left((s)^{n-1} \widetilde{\mathcal{M}}_{s}\left(\phi_{3}\right)(x)\right) d s d \nu\right]
\end{aligned}
$$

By a similar idea with the Duhamel principle for wave equations, the solution of the Cauchy problem for the nonhomogeneous biwave equation will be given at the next theorem

Theorem 4. The solution of the equation (1.1)-(1.2) takes the form $u=\widetilde{u}+v$, where $\widetilde{u}$ is the solution of the equation (2.1)-(2.2) and

$$
v(x, t)=\int_{0}^{t} \omega(x, t, \tau) d \tau
$$

where $\omega(x, t, \tau)$ is the solution of the homogeneous biwave equation

$$
\left(\frac{\partial^{2}}{\partial t^{2}}-a^{2} \Delta\right)\left(\frac{\partial^{2} \omega}{\partial t^{2}}-b^{2} \Delta \omega\right)=0, \quad t>\tau
$$

with the initial conditions

$$
\left.\omega\right|_{t=\tau}=0,\left.\quad \frac{\partial \omega}{\partial t}\right|_{t=\tau}=0,\left.\quad \frac{\partial^{2} \omega}{\partial t^{2}}\right|_{t=\tau}=0,\left.\quad \frac{\partial^{3} \omega}{\partial t^{3}}\right|_{t=\tau}=f(x, \tau) .
$$

Proof. We start with the observation that

$$
\begin{aligned}
& \frac{\partial^{4} v}{\partial t^{4}}-\left(a^{2}+b^{2}\right) \frac{\partial^{2}}{\partial t^{2}} \Delta v+a^{2} b^{2} \Delta^{2} v=f(x, t) \\
& \quad+\int_{0}^{t}\left(\frac{\partial^{4} \omega}{\partial t^{4}}-\left(a^{2}+b^{2}\right) \frac{\partial^{2}}{\partial t^{2}} \Delta \omega+a^{2} b^{2} \Delta^{2} \omega\right) d \tau=f(x, t) .
\end{aligned}
$$

Then, the above identity follows that

$$
\frac{\partial^{4} u}{\partial t^{4}}-\left(a^{2}+b^{2}\right) \frac{\partial^{2}}{\partial t^{2}} \Delta u+a^{2} b^{2} \Delta^{2} u=f(x, t) .
$$

Moreover,

$$
\begin{gathered}
\left.u\right|_{t=0}=\left.\widetilde{u}\right|_{t=0}+\left.v\right|_{t=0}=\phi_{0}(x)+0=\phi_{0}(x), \\
\left.\frac{\partial u}{\partial t}\right|_{t=0}=\left.\frac{\partial \widetilde{u}}{\partial t}\right|_{t=0}+\left.\frac{\partial v}{\partial t}\right|_{t=0}=\phi_{1}(x)+0=\phi_{1}(x), \\
\left.\frac{\partial^{2} u}{\partial t^{2}}\right|_{t=0}=\left.\frac{\partial^{2} \widetilde{u}}{\partial t^{2}}\right|_{t=0}+\left.\frac{\partial^{2} v}{\partial t^{2}}\right|_{t=0}=\phi_{2}(x)+0=\phi_{2}(x), \\
\left.\frac{\partial^{3} u}{\partial t^{3}}\right|_{t=0}=\left.\frac{\partial^{3} \widetilde{u}}{\partial t^{3}}\right|_{t=0}+\left.\frac{\partial^{3} v}{\partial t^{3}}\right|_{t=0}=\phi_{3}(x)+0=\phi_{3}(x) .
\end{gathered}
$$

So the theorem is proved. 


\section{Example}

Let us give an example demonstrating Theorem 1. Consider the equation

$$
\left(\frac{\partial^{2}}{\partial t^{2}}-\frac{\partial^{2}}{\partial x^{2}}\right)\left(\frac{\partial^{2} u}{\partial t^{2}}-\frac{1}{4} \frac{\partial^{2} u}{\partial x^{2}}\right)=0
$$

with the initial conditions

$$
\left.u\right|_{t=0}=0,\left.\quad \frac{\partial u}{\partial t}\right|_{t=0}=\sin x,\left.\quad \frac{\partial^{2} u}{\partial t^{2}}\right|_{t=0}=\cos x,\left.\quad \frac{\partial^{3} u}{\partial t^{3}}\right|_{t=0}=0 .
$$

The solution of the equation (3.1)-(3.2) is given by the formula

$$
u(x, t)=\frac{1}{3}\left(4 \cos \left(\frac{t}{2}\right) \cos x-4 \cos t \cos x-\left(-8 \sin \left(\frac{t}{2}\right)+\sin t\right) \sin x\right) .
$$

\section{References}

[1] X. Feng and M. Neilan. Finite element methods for a bi-wave equation modeling $d$-wave superconductors. J. Comput. Math., 28(3):331-353, 2010. http://dx.doi.org/10.4208/jcm.1001-m1001.

[2] X. Feng and M. Neilan. Discontinuous finite element methods for a bi-wave equation modeling d-wave superconductors. Math. Comput., 80(275):1303-1333, 2011. http://dx.doi.org/10.1090/S0025-5718-2010-02436-6.

[3] W.I. Fushchych, O.V. Roman and R.Z. Zhdanov. Symmetry reduction and some exact solutions of nonlinear biwave equations. Rep. Math. Phys., 37(2):267-281, 1996. http://dx.doi.org/10.1016/0034-4877(96)89767-9.

[4] J. Hadamard. Lectures on Cauchy's Problem in Linear Partial Differential Equations. Dover Publications, New York, 1953.

[5] R.B. Hetnarski and J. Ignaczak. Mathematical Theory of Elasticity. Taylor and Francis Books Inc., 2004.

[6] V. Korzyuk, O. Konopelko and E. Cheb. Boundary-value problems for fourthorder equations of hyperbolic and composite types. J. Math. Sci., 171:89-115, 2010. http://dx.doi.org/s10958-010-0128-2.

[7] V.I. Korzyuk and E.S. Cheb. The Cauchy problem for a fourthorder equation with a bi-wave operator. Differ. Equ., 43:688-695, 2007. http://dx.doi.org/S0012266107050126.

[8] N.I. Muskhelishvili. Some Basic Problems of the Mathematical Theory of Elasticity. Noordhoff International Publishing, 2010.

[9] K.K. Sabelfeld and I.A. Shalimova. Spherical Means for PDEs. VSP BV, 1997.

[10] A. Sommerfeld. Mechanics of Deformable Bodies. Lectures on Theoretical Physics, vol. II. Academic Press Inc., New York, 1950.

[11] E.M. Stein and R. Shakarchi. Fourier Analysis. An Introduction. Princeton Lectures in Analysis, 1. Princeton University Press, 2003.

[12] A. Torchinsky. The Fourier transform and the wave equation. ArXiv e-prints, April 2009. Available from Internet: http://arxiv.org/abs/0904.3252v1. 